\title{
Adopción de Buenas Prácticas Agrícolas (BPA): costo de cumplimiento y beneficios percibidos entre productores de fruta fresca
}

\author{
Adoption of Good Agricultural Practices (GAP): \\ cost of complying and perceived benefits by fresh \\ fruit producers \\ Gabriela Cofré ${ }^{1 *}$, Iván Riquelme ${ }^{1}$, Alejandra Engler ${ }^{1}$, Roberto Jara-Rojas ${ }^{1}$
}

\section{RESUMEN}

El comercio internacional de fruta fresca está regulado por distintos estándares y normativas, algunos de carácter obligatorio fiscalizados por entidades públicas, y otros de carácter voluntario desarrollados por cadenas de distribución de alimento mundial, como GlobalGap. En Chile, desde el año 2000 existen iniciativas de apoyo a la certificación de estándares privados de Buenas Prácticas Agrícolas (BPA) para facilitar el acceso a mercados más exigentes y competitivos. Este estudio de casos busca estimar costos de cumplimiento de BPA y su variabilidad, identificar beneficios y desventajas de la certificación. Se encuestaron 26 productores exportadores de fruta fresca de la zona central de Chile (Región de O’Higgins y del Maule), 12 certificados con estándares privados de BPA y 14 no certificados, pero que cumplen con el DS No 594 relacionado con condiciones sanitarias y ambientales básicas en los lugares de trabajo. A partir de los resultados, se determina que el costo de cumplimiento de BPA tiende a ser mayor en productor no certificado (\$308.095) que en certificado (\$166.105); la diferencia está marcada por el costo de implementación e influenciada por el tamaño predial. La principal ventaja al adoptar protocolos privados de BPA es acceder a más y mejores mercados $(>78 \%)$, pero además entregan seguridad a trabajadores agrícolas y consumidores finales. Los productores consideran como principal desventaja el incremento en los costos de producción (58,3\% certificado y $42,9 \%$ no certificado).

Palabras clave: BPA, costos de cumplimiento BPA, GlobalGap, calidad agroalimentaria.

\begin{abstract}
International commerce of fresh fruit is regulated by different standards and norms, some of which are obligatory and controlled by public entities and others which are voluntary, developed by global food distribution chains such as GlobalGap. In Chile, since the year 2000 there have been initiatives to support the certification of private GAP standards to facilitate access to the most competitive and demanding markets. This case study estimates the costs of GAP compliance and their variability, and identifies the benefits and disadvantages of certification. Twenty six fresh fruit exporters from the central zone of Chile (O'Higgins and Maule Regions) were interviewed, 12 certified by private GAP standards and 14 not certified but who comply with Chilean sanitary decree DS $N^{\circ} 594$, related to the basic safety and environmental standards in the workplace. The results show that the cost of GAP compliance tends to be greater for non-certified producers $(\$ 308,095$ Chilean pesos) than for certified producers $(\$ 166,105)$; the difference is produced by the implementation cost, and influenced by the size of the orchard. The main advantage to adopting private GAP protocols is the access to more and better markets (> 78\%); however they also provide security for agricultural workers and final consumers. Producers consider the main disadvantage to be the increase in production costs $(58.3 \%$ certified and $42.9 \%$ not certified).
\end{abstract}

Key words: GAP, costs of GAP compliance, GlobalGap, food quality.

\section{Introducción}

En las últimas décadas el comercio internacional de fruta fresca ha experimentado grandes cambios debido al creciente desarrollo de estándares y regulaciones sanitarias, fitosanitarios y de calidad, algunos de carácter obligatorio fiscalizados por entidades públicas, y otros de carácter voluntario desarrollados por las cadenas de distribución de alimento mundial $\mathrm{u}$ organizaciones internacionales. Dichos estándares surgen en respuesta a la exigencia de los consumidores por alimentos inocuos,

\footnotetext{
1 Departamento de Economía Agraria, Facultad de Ciencias Agrarias, Universidad de Talca, 2 Norte 685, Talca, Chile.

* gcofre@utalca.cl
}

Fecha de Recepción: 7 Julio, 2012.

Fecha de Aceptación: 14 Septiembre, 2012. 
saludables y amigables con el medio ambiente, lo que les permite manejar sus propias pautas y condiciones de producción (Henson, 2008; Amekawa, 2009; Bain, 2010; Henson et al., 2011). Ejemplo de estas cadenas son los supermercados TESCO, Carrefour, Walmart que exigen sus propios estándares de producción y sistemas de certificación de Buenas Prácticas Agrícolas (BPA) y trazabilidad a sus proveedores. El protocolo más requerido por los supermercados es GlobalGap e incluso es considerado como base para la implementación de otros protocolos privados de BPA como USAGap, TESCO Nature's Choice, Walmart (Amekawa, 2009; Bain, 2010; Fulponi, 2010; Henson et al., 2011). Estos basan su implementación en cuatro conceptos: (i) seguridad e inocuidad de los alimentos, (ii) protección al trabajador, (iii) protección al medio ambiente y (iv) bienestar animal. El énfasis dado en cada uno varía según protocolo, siendo no homologables entre ellos (Meléndez, 2005; Henson, 2008; Amekawa, 2009). Además están sujetos a actualizaciones, haciéndolos cada vez más estrictos al momento de acceder a estos canales de comercialización (Fulponi, 2010). Adicionalmente, la adopción de estos protocolos implica gastos asociados a implementación, mantención y certificación de BPA, que se adicionan a los costos de producción, lo que afecta la rentabilidad de los predios. De acuerdo a la literatura, los costos de adopción tienen un mayor impacto en los pequeños agricultores, debido a la escala de producción y falta de capacidad técnica o financiera para cumplir con la implementación y certificación de los protocolos privados, arriesgando incluso ser marginados del proceso exportador (Henson, 2008; Bain, 2010; Henson et al., 2011).

Chile, como principal exportador de fruta fresca a nivel mundial, ha mantenido desde el año 2000 políticas de apoyo a la certificación de BPA a través de programas de capacitación, asistencia y subsidios a las inversiones, especialmente a pequeños agricultores, logrando llegar en el año 2011 a 2.595 productores frutícolas certificados con GlobalGap de un total estimado de 7.800 productores (GLOBALG.A.P., 2011; Chilean Fresh Fruit Association, 2011). Sin embargo, a pesar del tiempo y éxito de las campañas de certificación, no existen estudios que estimen el impacto en costos para productores e identifiquen beneficios y limitaciones de la certificación. El objetivo de este trabajo es estimar costos de cumplimiento de BPA y su variabilidad, identificar beneficios y desventajas de estar "certificado", y factores intra y extraprediales que influyen en la decisión de implementar protocolos privados de BPA. Los resultados de este estudio serán de ayuda a las entidades públicas y privadas relacionadas con el sector frutícola exportador para el desarrollo de estrategias que motiven la adopción de protocolos privados de BPA entre los pequeños productores.

\section{Sector Frutícola Exportador Chileno}

Una ventaja comparativa de Chile son sus condiciones geográficas (Océano Pacífico y Cordillera de los Andes), que actúan como barrera natural contra la entrada de plagas y enfermedades (Agosin y Bravo, 2009). Su economía se basa en las exportaciones; $89,7 \%$ de los productos primarios son comercializados en el mercado internacional (ODEPA, 2010). El aporte del sector silvoagropecuario al Producto Interno Bruto (PIB) nacional es $3,8 \%$ en promedio en los últimos cinco años; $40 \%$ de éste proviene del sector frutícola (ODEPA, 2011). Alrededor de 75 especies de fruta son exportadas a más de 100 países (Agosin y Bravo, 2009), siendo las especies más transadas uva de mesa, manzanas, arándanos, paltas y cereza y los principales mercados de destino EE.UU. (36,1\%), Holanda (10,3\%), Reino Unido (5,1\%), España $(3,3 \%)$, Rusia $(3,9 \%)$, China $(1,1 \%)$, Hong Kong $(2,2 \%)$ y Taiwán $(2,4 \%)$ (ODEPA, 2011). Chile es un importante productor y exportador de fruta fresca en el Hemisferio Sur, exporta el 59,3\% del volumen total de la zona, siendo principal exportador de uva de mesa, ciruelas, arándanos, manzanas, duraznos, paltas y frutillas; segundo exportador de kiwis y tercero en peras (Tapia, 2006; Chilean Fresh Fruit Association, 2011).

En Chile instituciones públicas y privadas trabajan en conjunto generando programas e instrumentos de ayuda en la implementación de exigencias a productores y empresas exportadoras. Estos programas se han centrado en aspectos como: riego, manejo integrado de plagas (MIP), monitoreo de plagas, sistema de aseguramiento de calidad y BPA. Entre las entidades públicas, el Ministerio de Agricultura y el Servicio Agrícola y Ganadero (SAG) cumplen un rol importante en el proceso de certificación y negociación con nuestros socios comerciales alrededor del mundo, mientras que el sector privado está representado 
principalmente por la Asociación de Exportadores de Chile (ASOEX) y la Federación de Productores de Fruta de Chile (FEDEFRUTA). Dentro de las principales iniciativas públicas está la Comisión Nacional de Buenas Prácticas Agrícolas (2001), cuyo objetivo es asesorar al Ministerio de Agricultura en la formulación de políticas destinadas a incorporar y difundir el concepto de BPA dentro de los procesos productivos agropecuarios (SAG, 2012). Adicionalmente, la Fundación para el Desarrollo Frutícola (FDF), entidad privada que tiene como objetivo fomentar el desarrollo del sector frutícola, lanza en 2003 "ChileGap", un protocolo cuyo objetivo es homologar las exigencias de GlobalGap, Davis Fresh y USAGap para disminuir los costos de certificación e inspecciones de fruta que va a mercados con diferentes exigencias de protocolos (ODEPA, 2004; Chilean Fresh Fruit Association, 2011). Por otra parte, en Chile se exige el Decreto Supremo $\mathrm{N}^{\circ} 594$, que indica las normas a seguir por las empresas con respecto al cumplimiento de las condiciones sanitarias y ambientales que resguarden la salud y bienestar de los trabajadores (Biblioteca del Congreso Nacional de Chile, 2012); además existe una resolución de carácter obligatorio ( $\mathrm{N}^{\circ} 3410 / 2002$ del SAG) que exige la implementación de BPA a todos aquellos productores que produzcan y exporten las especies que están bajo esta resolución (ODEPA, 2004). En general, el sector frutícola desde principios de 2000 hasta la fecha ha invertido grandes esfuerzos en posicionarse en los mercados agroalimentarios internacionales, construyendo una imagen positiva basada en la calidad, inocuidad y protección del medio ambiente. Actualmente, se está desarrollando una fuerte estrategia de marketing impulsando una marca sectorial como país, "Fruits from Chile", orientada a consolidar el sector frutícola en el mercado mundial, basado en la calidad (alimentos inocuos), origen (clima y geografía) y temple de la industria y sus trabajadores (ProChile, 2012).

\section{Metodología}

\subsection{Selección de zona de estudio y productores frutícolas}

La estimación y comparación de costos relacionados con la implementación, mantención y certificación de BPA se abordó a través de una metodología de estudio de casos realizada en la zona central de Chile, entre las regiones de O'Higgins y del Maule ( $\left.33^{\circ} 50^{\prime}-36^{\circ} 33^{\prime}\right)$, zonas que concentran el $45 \%$ de la superficie nacional de frutales (ODEPA, 2011). Los productores tenían como único requisito producir fruta fresca de exportación, independiente de la especie. Se trabajó con 26 productores, 12 "certificados" que han adoptado algún protocolo privado de BPA como por ejemplo GlobalGap, y 14 "no certificados", es decir, aquellos que no han adoptado estos protocolos, pero cumplen el D.S $\mathrm{N}^{\circ} 594$ que exige Chile. Los principales frutales cultivados por los productores eran: arándano, cereza, ciruela, durazno, frutilla, kiwi, manzana, pera y uva de mesa.

\subsection{Levantamiento de información}

La información requerida para este estudio fue obtenida mediante una encuesta aplicada a productores durante marzo-abril de 2011. El cuestionario está compuesto por cuatro secciones: i) información del productor, donde se consideraban aspectos como edad del productor, nivel educacional, años de experiencia en el sector y participación en asociaciones gremiales; $i i)$ estructura productiva del predio, donde se consultaba superficie total del predio y especies frutales cultivadas; iii) comercialización y certificación de BPA, en esta sección se preguntaba mercado de destino, forma de comercialización y protocolos privados de certificación de BPA implementados en el predio; iv) cumplimiento de estándares sanitarios y fitosanitarios para la exportación, se consultaba por costos de inversión, mantención y manejo de registros para las BPA. Además se incluyeron preguntas de percepción para determinar los beneficios que implica implementar protocolos privados de BPA en el predio. En esta última pregunta se utilizó una escala de Likert que consideraba cinco categorías, desde Muy de acuerdo hasta Muy en desacuerdo, para cada ítem consultado: mercado-precio, trabajadores agrícolas, manejo de huerto y costos de producción, rentabilidad del huerto y, por último, beneficios al consumidor. La encuesta se aplicó mediante entrevista personal. Al final de ésta se realizaron algunas preguntas abiertas relacionadas con las ventajas y desventajas de estar certificado con algún protocolo privado de certificación de BPA. Los análisis realizados fueron de tipo descriptivo y de frecuencia, y se realizaron mediante el programa estadístico SPSS 15.0. 


\section{Resultados y Discusión}

\subsection{Descripción de productores, sistemas productivos frutícolas y estrategias de comercialización}

Entre las principales características de los productores se puede mencionar que tanto productores "certificados" como "no certificados" presentan una edad promedio similar cercana a 55 años, con un rango entre 28 y 93 años. El nivel educacional del $100 \%$ de los productores "certificados" es de nivel superior (universitario o técnico), pero en el caso de "no certificados", el 71,4\% sólo tiene educación media y 14,3\%, básica. Ambos grupos de productores tienen una experiencia en el rubro de 25 años, con un rango entre 5 y 50 años. El 41,7\% de los productores "certificados" participa en alguna asociación gremial, ya sea ASOEX, FEDEFRUTA, o algún comité de fruta (kiwi, arándanos); en productores "no certificados" este porcentaje sólo alcanza al 7,1\%. Estos resultados sugieren que el nivel educacional y la participación en agrupaciones gremiales son un factor relevante ante la decisión de adoptar e implementar protocolos privados de certificación. Las principales asociaciones gremiales realizan grandes esfuerzos entre sus asociados para implementar certificaciones privadas de BPA. Trabajos elaborados por diversos autores indican que la adopción de estos protocolos está favorecida por factores como tamaño predial, participación en organizaciones gremiales, nivel educacional, acceso a asistencia técnica y financiera (Henson, 2008; Nahuelhual et al., 2009; Henson et al., 2011; Herzfeld et al., 2011).

En relación con los sistemas productivos (Tabla 1), productores "certificados" tienen mayor superficie predial en comparación con productores "no certificados", en promedio 163,1 y 42,1 hectáreas respectivamente, sugiriendo al igual que en otros estudios que la certificación de BPA o sistemas de producción limpia está asociada preferentemente a predios de mayor tamaño, por el nivel de inversión requerido (Henson, 2008; Nahuelhual et al., 2009). Los productores "certificados" destinan mayor superficie de sus predios a plantación de frutales $(86,9 \%)$, y tienen un mayor número de especies, en promedio cuatro especies frutales diferentes. En cambio, el productor "no certificado" destina sólo el $68,6 \%$ de la superficie total a la producción

Tabla 1. Descripción de productores y sistemas productivos frutícolas en predios "certificados" y "no certificados" con protocolos privados de Buenas Prácticas Agrícolas.

\begin{tabular}{lcc}
\hline Características & \multicolumn{2}{c}{ Productores frutícolas $(\mathrm{n}=26)$} \\
\cline { 2 - 3 } Edad (años) & Certificados (n=12) & No certificados $(\mathrm{n}=14)$ \\
\hline Nivel educacional & 54 & 56 \\
$\quad$ Superior & $(28-93)$ & $(35-84)$ \\
$\quad$ Media & $100 \%$ & $71,4 \%$ \\
Básica & $0 \%$ & $14,30 \%$ \\
\hline Experiencia sector frutícola (años) & $0 \%$ & $14,30 \%$ \\
\hline Participación en asociación gremial & 25 & 27 \\
\hline Superficie total predio (ha) & $(5-50)$ & $(5-50)$ \\
\hline Superficie frutal total (ha) y participación en la superficie total del predio $(\%)$ & $41,70 \%$ & $7,10 \%$ \\
\hline Participación superficie especie frutal principal, sobre superficie frutal total $(\%)$ & $161,8(86,9 \%)$ & 42,1 \\
\hline No de especies frutales en el predio & $(12-450)$ & $(3,6-120)$ \\
\hline
\end{tabular}

Nota: en paréntesis aparece el rango de la variable. 
de frutales, cultivando en promedio dos especies, donde la más rentable ocupa el 52,2\% promedio de la superficie total de frutales. Ambos grupos destinan el resto del predio al cultivo de otros rubros, los más relevantes cereales, hortalizas, semilleros y vides viníferas. Como estrategia comercial, la mayoría de los productores, con y sin certificación, comercializan su fruta con dos empresas exportadoras en promedio. El $66,7 \%$ y $71,4 \%$ de los productores "certificados" y "no certificados", respectivamente, solicita avances en dinero a las empresas exportadoras. Una vez entregada la fruta a la empresa, los retornos económicos son pagados a los productores mediante liquidaciones, el período de tiempo que demora este proceso es de 4 a 5 meses, independiente de si es un productor certificado o no. A partir de los resultados, se observa que un productor "certificado" tiende a cultivar un mayor número de especies frutales, y que, a mayor superficie frutal, los productores comercializan con un mayor número de empresas exportadoras, reflejando que, a mayor tamaño predial, mayor es la capacidad técnico-económica del productor, favoreciendo su capacidad competitiva dentro del sector exportador.

\subsection{Certificación protocolos privados BPA}

El 100\% de los productores "certificados" tienen implementado el protocolo GlobalGap; el $50 \%$ tiene adicionalmente TESCO y el $16,6 \%$ USAGap. La adopción de certificaciones privadas de BPA en estos productores comenzó en el año 2001 con GlobalGap, seguido en 2005 con TESCO y en 2009 con USAGap. Los productores TESCO tienen mayor superficie certificada (150,8 ha) en comparación con aquellos que han implementado sólo GlobalGap (132,7 ha) y USAGap (80,7 ha), como se muestra en la Tabla 2. Los resultados avalan la tendencia mundial, donde GlobalGap es considerado el principal sistema de aseguramiento de calidad a nivel de producción primaria, y es la base para la implementación de otros protocolos privados de certificación (Amekawa, 2009; Bain, 2010). El número promedio de especies frutales certificadas por productor varía entre cuatro y cinco, independiente del protocolo certificado.

\subsection{Costos certificación privados de BPA}

La implementación de certificaciones privadas de BPA no sólo involucra costos asociados con la obtención del certificado (auditoría de certificación), sino aquellos relacionados con implementación, mantención y manejo de registro de BPA. En Chile todos los productores deben cumplir el DS N ${ }^{\circ} 594$, que obliga la implementación de medidas de seguridad e infraestructura adecuadas para el cumplimiento de las condiciones sanitarias y ambientales básicas en los predios.

El costo de implementación, mantención y manejo de registro de BPA por hectárea tiende a ser mayor en productores "no certificados" como se muestra en la Tabla 3. El costo que marca la diferencia entre ambos grupos es implementación; para un productor "no certificado" el costo es de $\$ 260.636$ en promedio por hectárea, y para un productor "certificado" de \$ 115.196. Sin embargo, es importante considerar que el costo de implementación entre productores "certificados" y "no certificados" tiene un amplio rango que va desde los \$ 13.343 hasta los $\$ 760.000$. En cuanto a mantención de BPA, el costo es similar entre los productores "certificados"

Tabla 2. Característica de los productores "certificados" con los principales protocolos privados de Buenas Prácticas Agrícolas.

\begin{tabular}{lccc}
\hline \multirow{2}{*}{ Característica } & \multicolumn{2}{c}{ Protocolos privados de certificación BPA } \\
\cline { 2 - 4 } & GlobalGap & TESCO & USAGap \\
\hline Primera certificación (año) & 2001 & 2005 & 2009 \\
\hline $\mathrm{N}^{\mathrm{o}}$ años certificado & $(2001-2010)$ & $(2005-2010)$ & $(2009-2010)$ \\
\hline $\mathrm{N}^{\mathbf{o}}$ especies frutales certificadas & 5,5 & 4 & 1,5 \\
\hline Superficie frutal certificada (ha) & $(1-10)$ & $(1-6)$ & 4 \\
\hline
\end{tabular}

Nota: en paréntesis aparece el rango de la variable. 
Tabla 3. Inversión en implementación, y costos de mantención y manejo de registros de Buenas Prácticas Agrícolas por hectárea, en productores "certificados" y "no certificados".

\begin{tabular}{lcc}
\hline \multirow{2}{*}{ Buenas Prácticas Agrícolas (BPA) } & \multicolumn{2}{c}{ Productores Frutícolas $(\mathrm{n}=26)$} \\
\cline { 2 - 3 } & "Certificados” $(\mathrm{n}=12)$ & "No Certificados” $(\mathrm{n}=14)$ \\
\hline Implementación (\$/ha) & 115.196 & 260.636 \\
& $(13.343-558.333)$ & $(28.986-760.000)$ \\
\hline Mantención (\$/ha) & 47.072 & 40.437 \\
\hline Manejo de Registro (\$/ha) & $(5.556-127.000)$ & $(7.167-108.889)$ \\
\hline Total (\$/ha) & 3.837 & 7.022 \\
& $(667-17.500)$ & $(250-34.400)$ \\
\hline
\end{tabular}

Nota: en paréntesis aparece el rango de la variable.

y "no certificados", con valores promedio cercanos a \$ 44.000 por hectárea; en este ítem también se observa un amplio rango entre $\$ 5.556-\$ 127.000$. El costo de manejo de registro de BPA promedio por hectárea en productores "certificados" es de \$ 3.837 y en "no certificados" de \$ 7.022. Considerando todos los ítems anteriores, se puede decir que el costo total de cumplimiento de BPA por hectárea para un productor "certificado" es de \$ 166.105 variando entre $\$ 21.778$ y $\$ 600.833$, y para un productor "no certificado", este costo es de $\$ 308.095$ con un rango entre \$ 89.821 y $\$ 845.200$. La diferencia entre productores está marcada principalmente por el costo de implementación, ya que en el resto de los ítems, mantención y manejo de registro, el costo promedio es similar entre productor "certificado" y "no certificado". Esta variabilidad podría ser explicada por el tamaño predial; la tendencia es que a mayor superficie frutal, el costo total de cumplimiento de BPA por superficie disminuye. Lo anterior se refleja en productores "no certificados", los que al tener menor superficie frutal en comparación con productores "certificados" asumen un mayor costo de cumplimiento de BPA, siendo relevantes las economías de escala. Los resultados obtenidos avalan a autores como Henson (2008), quien menciona que los costos asociados a protocolos privados de certificación obstaculizan el comercio de fruta fresca, siendo más afectados países en vías de desarrollo que no cuentan con recursos suficientes en capital humano, tecnología e infraestructura para el aseguramiento de la calidad, por lo tanto, las grandes exportadoras y/o productores por su tamaño (economía de escala) pueden amortizar los costos de cumplimiento asociados a los protocolos privados de certificación de BPA.
En cuanto al costo de certificación, el valor promedio anual es de $\$ 540.500$, fluctuando entre $\$ 300.000$ y $\$ 1.000 .000$ (Tabla 4). Este valor no depende del tamaño predial, ni la especie frutal a certificar, sino del número de auditorías necesarias para cumplir con este procedimiento. Algunos protocolos de certificación, por ejemplo GlobalGap, se pueden realizar en forma grupal o individual. $\mathrm{Al}$ tomar la primera opción el costo es menor, siendo una posibilidad para el pequeño productor.

Para un productor "certificado", el costo de certificación representa en promedio el $25,7 \%$ del costo total de BPA anual (mantención y manejo de registro), variando entre $4,7 \%$ y $61,1 \%$. Este porcentaje dependerá del tipo de certificación, grupal o individual y del tamaño predial. Como ya se ha mencionado, a mayor superficie frutal, el costo de certificación por hectárea disminuirá.

Tabla 4. Costo anual de certificación de protocolos privados de Buenas Prácticas Agrícolas, GlobalGap, TESCO, USAGap.

\begin{tabular}{lc}
\hline $\begin{array}{l}\text { Protocolo Privado de } \\
\text { Certificación BPA }\end{array}$ & Costo certificación (\$/anual) \\
\hline GlobalGap & 550.000 \\
\hline TESCO & $(1.000 .000-320.000)$ \\
\hline USAGap & 522.000 \\
\hline Total & $(1.000 .000-300.000)$ \\
\hline
\end{tabular}

Nota: en paréntesis aparece el rango de la variable. 


\subsection{Percepción de beneficios y dificultades de estar certificado con algún protocolo privado de Buenas Prácticas Agrícolas}

La Tabla 5 muestra los beneficios y desventajas de estar certificado con BPA, los resultados muestran que productores "certificados" y "no certificados" consideran como principal ventaja acceder a más y mejores mercados, $81,7 \%$ y $78,6 \%$, respectivamente. Lo anterior, coincide con el 45,5\% de los productores "certificados" que adoptaron motivados por acceder al mercado, el otro 45,5\% atribuye su adopción a exigencia de la empresa exportadora, y sólo el 9\% a mayores precios de venta. Otros beneficios atribuidos a la certificación se relacionan con el manejo de huerto y seguridad de trabajadores agrícolas y consumidores. El $80 \%$ de los productores encuestados considera que la adopción de protocolos privados de BPA reduce el riesgo de enfermedades e intoxicaciones relacionadas con agroquímicos en trabajadores agrícolas, que el sistema de trazabilidad entrega seguridad a los consumidores, y además el manejo de registros permite un buen control de inventario en insumo y agroquímicos, siendo una herramienta útil en la administración del predio. Estos resultados avalan las conclusiones de distintos autores (Amekawa, 2009; Bain, 2010; Henson et al., 2011), quienes mencionan que la adopción de protocolos privados de BPA permite a las empresas exportadoras y/o productores acceder a mayor $\mathrm{y}$

Tabla 5. Percepción de beneficio y desventaja de la implementación de protocolos privados de certificación de Buenas Prácticas Agrícolas.

\begin{tabular}{|c|c|c|c|c|c|c|}
\hline \multirow[t]{2}{*}{ Ítem } & \multicolumn{3}{|c|}{$\begin{array}{l}\text { Productores "Certificados" }(\%) \\
\qquad(\mathrm{n}=12)\end{array}$} & \multicolumn{3}{|c|}{$\begin{array}{l}\text { Productores "No certificados" }(\%) \\
\qquad(\mathrm{n}=14)\end{array}$} \\
\hline & Desacuerdo & Neutro & De acuerdo & Desacuerdo & Neutro & De acuerdo \\
\hline \multicolumn{7}{|l|}{ Mercado y Precio } \\
\hline $\begin{array}{l}\text { 1. La certificación permite acceder a } \\
\text { más y mejores mercados }\end{array}$ & 0 & 8,3 & 91,7 & 0 & 21,4 & 78,6 \\
\hline $\begin{array}{l}\text { 2. La certificación mejora los retornos } \\
\text { en la venta de fruta }\end{array}$ & 16,7 & 16,7 & 66,7 & 7,1 & 14,3 & 78,6 \\
\hline \multicolumn{7}{|l|}{ Beneficios Trabajadores Agrícolas } \\
\hline $\begin{array}{l}\text { 3. La certificación disminuye el riesgo } \\
\text { de intoxicaciones y enfermedades } \\
\text { relacionadas con agroquímicos }\end{array}$ & 8,3 & 0 & 91,7 & 0 & 7,1 & 92,9 \\
\hline \multicolumn{7}{|l|}{ Manejo de Huerto y Costos de Producción } \\
\hline $\begin{array}{l}\text { 4. El programa fitosanitario permite } \\
\text { obtener un producto de mayor } \\
\text { calidad }\end{array}$ & 8,3 & 8,3 & 83,3 & 0 & 7,1 & 92,9 \\
\hline $\begin{array}{l}\text { 5. La implementación de BPA no } \\
\text { implica mayores costos de } \\
\text { producción }\end{array}$ & 58,3 & 25 & 16,7 & 42,9 & 14,3 & 42,9 \\
\hline $\begin{array}{l}\text { 6. El manejo de registros permite mejor } \\
\text { control del stock de agroquímicos y } \\
\text { gestión empresarial del predio }\end{array}$ & 8,3 & 0 & 91,7 & 7,1 & 7,1 & 85,7 \\
\hline \multicolumn{7}{|l|}{ Beneficios para el Consumidor } \\
\hline $\begin{array}{l}\text { 7. La trazabilidad y análisis del } \\
\text { producto (residuos) es un seguro } \\
\text { efectivo para el consumidor }\end{array}$ & 0 & 0 & 100 & 0 & 7,1 & 92,9 \\
\hline \multicolumn{7}{|l|}{ Productividad y Rentabilidad del Huerto } \\
\hline $\begin{array}{l}\text { 8. La certificación permite aumentar la } \\
\text { rentabilidad del predio }\end{array}$ & 25 & 0 & 75 & 14,3 & 21,4 & 57,1 \\
\hline
\end{tabular}


mejores mercados. En cambio, productores "no certificados", generalmente pequeños productores, son excluidos de los mercados más exigentes teniendo sólo la posibilidad de optar a mercados de menor requerimiento. En relación con los beneficios en retorno, costo y rentabilidad los productores tienen distintas percepciones. Mientras que el 66,7\% de productores "certificados" considera que los retornos son mayores al adoptar una certificación, esta cifra sube al 78,5\% en el grupo "no certificado", lo que revela que existe una percepción de mayor retorno entre quienes no han tenido la posibilidad de evaluarlos anteriormente. De la misma manera, la percepción respecto a costos de mantención de BPA y rentabilidad de la certificación también varía entre el grupo de "certificados" y "no certificados". En relación a los costos de producción, el 16,7\% de los productores "certificados" consideran que estos aumentan, y un 42,9\% tiene la misma percepción en productores "no certificados". Con respecto a rentabilidad, el $75 \%$ y $57,1 \%$ de los productores "certificados" y "no certificados", respectivamente, consideran que ésta aumenta con la adopción de los protocolos de certificación. Esto permite concluir que, en general, el costo que se asume para certificarse no es mayor, lo que este estudio avala, $\mathrm{y}$, por otra parte, sí se considera que genera una rentabilidad positiva. Por tanto, la no certificación de productores estaría dada por limitantes como capacidad de gestión o barreras de financiamiento a la inversión, donde este estudio ha demostrado que existen diferencias entre "certificados" y "no certificados".

Los resultados de percepción muestran que productores "no certificados" reconocen los beneficios de adoptar protocolos de certificación, como son mayor y mejor acceso al mercado, manejo de huerto, incremento retorno productor, y seguridad para trabajadores y/o consumidores. La principal desventaja para este grupo es el aumento en los costos y reducción de la rentabilidad. Ellos cumplen con condiciones sanitarias y de seguridad ( $\mathrm{DS} \mathrm{N}^{\circ}$ 594); avanzar a la etapa de certificación les implica asumir nuevas inversiones en infraestructura, teniendo ya por este concepto un costo elevado en comparación con los "certificados"; además, el costo de certificación puede llegar a representar el 25,7\% del costo de mantención de BPA anual. Dada la característica de pequeño productor, amortizar estos nuevos costos es para ellos un desafío, ya que cuentan con una estructura productiva reducida, caracterizada por predios de baja superficie, en algunos casos bajo nivel educacional y poca participación en asociaciones, factores que favorecen la baja adopción de estas prácticas. Los productores "certificados", en general, se muestran conformes con la adopción de protocolos privados de BPA, pero consideran que afecta su estructura de costo (58,3\%), y un $25 \%$ de ellos no aprecian mejores retornos.

\section{Conclusiones}

A partir del estudio de caso, se determina que el costo de cumplimiento de BPA tiende a ser mayor en productores "no certificados" (\$ 308.095), que en "certificados" (\$166.105). Esta diferencia está marcada principalmente por el costo de implementación e influenciada por el tamaño predial. La tendencia es a mayor superficie menor es el costo de implementación de BPA por hectárea. Además, el costo de certificación representa un $25,7 \%$ de los costos de mantención de BPA anual, variando entre 4,7 y $61,1 \%$. Todos los productores, certificados o no, consideran que la principal ventaja al adoptar protocolos privados de BPA es acceder a más y mejores mercados (>78\%), pero además entregan seguridad a trabajadores agrícolas y consumidores finales, a través del buen manejo de insumos y agroquímicos y sistemas de trazabilidad. La principal desventaja es el incremento de los costos de producción, $58,3 \%$ productor "certificado y $42,9 \%$ productor "no certificado".

\section{Agradecimientos}

Esta investigación fue financiada por el proyecto FONDECYT No 1100063. 


\section{Referencias}

Agosin, M.; Bravo, C.

2009 The emergence of new successful export activities in Latin America: the case of Chile. Research Network Paper \# R-552. Inter-American Development Bank.

Amekawa, Y.

2009 Reflections on the Growing Influence of Good Agricultural Practices in the Global South. Journal of Agricultural and Environmental Ethics, 22, 531-557.

Bain, C.

2010 Governing the Global Value Chain: GLOBALGAP and the Chilean Fresh Fruit Industry, 1-23.

Biblioteca del Congreso Nacional de Chile

2012 Aprueba reglamento sobre condiciones sanitarias y ambientales básicas en los lugares de trabajo. Recuperado el 12 de junio de 2012, de http://www.leychile.cl/ Navegar?idNorma $=167766$

Chilean Fresh Fruit Association

2011 Chileanfreshfruit. Recuperado el 14 de diciembre de 2011, de chileanfreshfruit: http://www.chileanfreshfruit. com/newchileanfreshfruit/pdf/cff_espa\%C3\%B1ol.pdf

Fulponi, L.

2010 Impacto de los estándares y regulaciones sobre las cadenas agroexportadoras a nivel mundial. Impacto de las Regulaciones y Normas Internacionales de Calidad (págs. 1-31). Talca.

GLOBALG.A.P.

201. Globalg.A.P.Annual Report 2011. Germany: GLOBALG.A.P.

Henson, S.

2008 The role of public and private standards in regulating international food markets. Journal of International Agricultural Trade and Development, 4 (1), 63-81.

Henson, S.; Masakure, O. y Cranfield, J.

2011 Do Fresh Produce Exporters in Sub-Saharan Africa Benefit from GlobalGAP Certification? World Development, 375-386.

Herzfeld, T.; Drescher, L. S. y Grebitus, C.

2011 Cross-national adoption of private food quality standards. Food Policy, 36, 401-411.
Meléndez, L.

2005 Comparación entre sistemas de certificaciones de Buenas Prácticas Agrícolas. Temuco: Instituto Nacional de Investigación Agropecuaria (INIA).

Nahuelhual, L.; Engler, A.; Carrillo, B.; Moreira, V. y Castro, I. 2009 Adoption of cleaner production practices by dairy farmers in southern Chile. Ciencia e Investigación Agraria, $36(1), 97-106$.

ODEPA

2011 Oficina de Estudios y Políticas Agrarias. Recuperado el 14 de diciembre de 2011, de http://www.odepa.gob.cl/ menu/ComercioExterior.action;jsessionid=8D942C1924C 0C67414DE4D54ECD9A2C5.

ODEPA

2010 Evolución de las exportaciones silvoagropecuarias de Chile a sus socios comerciales. Santiago-Chile: Oficina de Estudios y Políticas Agraria (ODEPA).

ODEPA

2004 Temporada Agrícola No 23. Santiago-Chile: ODEPA. ProChile

2012 Dirección de Promoción de Exportaciones. Recuperado el 10 de febrero de 2012, de http://rc.prochile.gob.cl/ noticia/29971/2.

SAG

2012 Servicio Agrícola y Ganadero. Recuperado el 10 de junio de 2012, de Buenas Prácticas Agrícolas: http://www. sag.cl/openDocs/asp/pagDefault.asp?boton=Doc54\&argI nstanciaId=54\&argCarpetaId=1437\&argTreeNodosAbier tos $=(1437)(-54) \& \arg$ TreeNodoActual $=1437 \& \arg$ TreeNo doSel $=$

Tapia, L.

2006 Estimación de la competitividad de los países exportadores de fruta del Hemisferio Sur a partir del índice de competitividad para el crecimiento en el período 2001-2004. IDESIA, 49-59. 
